\title{
Coupled Diffusion in Slags ${ }^{*}$
}

\author{
By $W-K . L U^{* *}$
}

\section{Synopsis}

Multiple-component diffusion in slags is investigated theoretically in terms of Irreversible Thermodynamics. The validity of linear phenomenological equations is assumed and the generalized forces are defined as the negative gradients of electrochemical potentials. The relationship among the fluxes due to the assumption of the quasi-steady state of electrical charge transfer leads to the expression of electrical field as a function of gradients of chemical potentials and transference numbers. The final flux equations are shown to be functions of gradients of chemical potentials, transference numbers and, of course, phenomenological coefficients. Their applications and the limiting case, under equilibrium or no fux conditions, are also discussed.

\section{Introduction}

Guggenheim $^{1)}$ suggested that electrochemical potential, $\bar{\mu}_{i}$, should be used in the equation which defines Gibbs free energy whenever an ionic system is under consideration.

$$
d G=-S d T+V d p+\sum_{i} \bar{\mu}_{i} d n_{i}
$$

The thermodynamic definition of the electrochemical potential may be defined in the following way. For two phases, $\alpha$ and $\beta$, of identical chemical composition, the difference in electrochemical potential is

$$
\bar{\mu}_{i}^{(\beta)}-\bar{\mu}_{i}^{(\alpha)}=z_{i} F\left(\psi^{(\beta)}-\phi^{(\alpha)}\right)
$$

where $\psi^{(\beta)}-\psi^{(\alpha)}$ is the electrical potential difference between the two phases. For the distribution of the ionic species $i$ between two phases, $\alpha$ and $\beta$, of different chemical composition the equilibrium condition is equality of the electrochemical potential, that is to say,

$$
\bar{\mu}_{i}^{(\alpha)}=\bar{\mu}_{i}^{(\beta)} .
$$

Any splitting of $\bar{\mu}_{i}^{(\beta)}-\bar{\mu}_{i}^{(\alpha)}$ into a chemical part and an electrical part is in general arbitrary, for example,

$$
\bar{\mu}_{i}^{(\beta)}-\bar{\mu}_{i}^{(\alpha)}=\mu_{i}^{(\beta)}-\mu_{i}^{(\alpha)}+z_{i} F\left(\phi^{(\beta)}-\phi^{(\alpha)}\right) .
$$

Based on the principles of Irreversible Thermodynamics diffusional flux in an isothermal, isobaric and continuous system of $\mathcal{N}+1$ components may be expressed as follows:

$$
J_{i}=\sum_{k=0}^{N} L_{i k}^{\prime} X_{k}^{\prime}, \quad i=0,1,2,3, \ldots \mathcal{N} .
$$

When a specific frame of reference is chosen only $\mathcal{N}$ flux equation in Eq. (5) are independent. For simplicity, we put the spacial co-ordinate system on the solvent in dilute solutions or on the relatively immobile polymers, e.g., the silicate ions in molten slags $^{4)}$ and name this chemical species the $0^{\text {th }}$ component. Then Eq. (5) becomes

$$
J_{i}=\sum_{k=0}^{N} L_{i k}^{\prime} X_{k}^{\prime}, \quad i=1,2,3, \ldots \mathcal{N}
$$

and $J_{0}=0$.

For systems of non-charged particles, the generalized force $X_{k}^{\prime}$ is the negative gradient of the chemical potential of the $k^{t h}$ species. It may be assumed that the dominant interaction between charged particles in ionic solutions is through the interaction with and the contribution to the overall environment, electrical field. Following de Groot $^{2)}$ such electrical effects can be accounted for by selecting the generalized force, $X_{k}^{\prime \prime}$, as the negative gradient of electrochemical potential of $k^{t h}$ species.

$$
J_{i}=\sum_{k=0}^{N} L_{i k}^{\prime \prime} X_{k}^{\prime \prime}, \quad i=1,2,3, \ldots \mathcal{N}
$$

The Gibbs-Duhem equation in terms of the electrochemical potential can be used to eliminate one of $X^{\prime \prime}$ 's. After substituting

$$
\frac{\partial \bar{\mu}_{0}}{\partial X}=-\sum_{k=1}^{N} \frac{C_{k}}{C_{0}} \frac{\partial \bar{\mu}_{k}}{\partial X}
$$

into Eq. (6),

$$
J_{i}=\sum_{k=1}^{N} L_{i k} X_{k}
$$

where

$$
\begin{gathered}
X_{k}=-\frac{\partial \bar{\mu}_{k}}{\partial X}=X_{k}^{\prime \prime} \\
L_{i k}=L_{i k}^{\prime \prime}-L_{i k}^{\prime \prime} \frac{C_{k}}{C_{0}} .
\end{gathered}
$$

This type of formulation with various simplifying assumptions has been proved to be successful in the interpretation of experimental results of diffusion in molten silicates. ${ }^{4}$ This communication is intended to study Eq. (6) with less restrictive assumptions than previously employed.

Besides all the assumptions which are necessary in order to obtain Eq. (6), such as the adequacy of linear phenomenological equations and the selection of frame of reference, the additional one is given as Eq. (7).

$$
\sum_{i=1}^{N} Z_{i} J_{i}=0 \quad \text { and } \quad J_{0}=0
$$

* Received July 11, 1970.

** Department of Metallurgy and Materials Science, McMaster University, Hamilton, Ontario, Canada. 
It means that the net accumulation of the electrical charge in any volume element due to diffusion is zero. This is a weaker assumption than electroneutrality. The system under consideration at the moment is not subject to the external electrical field; Eq. (7) is needed to express the electrical portion of the generalized force in terms of the chemical driving force.

\section{Derivation}

Rewrite Eq. (6a) with the splitting of $X_{k}$ into a chemical part and an electrical part according to Eq. (4).

$$
\begin{array}{r}
J_{i}=-\sum_{k=1}^{N} L_{i k} \frac{\partial \mu_{k}}{\partial X}-\frac{\partial \psi}{\partial X} F \sum_{k=1}^{N} Z_{k} L_{i k} \\
i=1,2,3, \ldots \mathcal{N} \ldots \ldots \ldots \ldots \ldots
\end{array}
$$

Substituting Eq. (6b) into Eq. (7),

$$
\sum_{i, k=1}^{N} Z_{i} L_{i k} \frac{\partial \mu_{k}}{\partial X}+F \frac{\partial \psi}{\partial X} \sum_{i, k=1}^{N} Z_{i} Z_{k} L_{i k}=0
$$

By re-arranging the above equation, we obtain

$$
\frac{\partial \psi}{\partial X}=\frac{-\sum_{i, k=1}^{N} Z_{i} L_{i k} \frac{\partial \mu_{k}}{\partial X}}{F \sum_{i, k=1}^{N} Z_{i} Z_{k} L_{i k}} .
$$

Multiply the numerator and denominator both by $\partial \psi / \partial X \cdot F$ and carry out the double summation over index $i$ first, then it becomes

$$
\begin{gathered}
\frac{\partial \psi}{\partial X}=\frac{-\sum_{k=1}^{N} \frac{\partial \mu_{k}}{\partial X_{i}} \sum_{i=1}^{N} F Z_{i} L_{i k}\left(\frac{\partial \psi}{\partial X}\right)}{\sum_{k=1}^{N} F Z_{k} \sum_{i=1}^{N} F Z_{i} L_{i k}\left(\frac{\partial \psi}{\partial X}\right)} \\
\frac{\partial \psi}{\partial X}=-\frac{1}{F} \sum_{k=1}^{N} \frac{\partial \mu_{k}}{\partial X} \frac{1}{Z_{k}}\left[\frac{Z_{k} J_{k}^{(0)}}{\sum_{k=1}^{N} Z_{k} J_{k}^{(0)}}\right] \cdots \cdots
\end{gathered}
$$

where

$$
J_{k}^{(0)}=F \sum_{i=1}^{N} Z_{i} L_{k i} \frac{\partial \psi}{\partial X}
$$

From Onsager reciprocity theorem

$$
J_{k}^{(0)}=F \sum_{i=1}^{N} Z_{i} L_{i k} \frac{\partial \psi}{\partial X} .
$$

It is easy to see from Eq. (6b) that $J_{k}^{(0)}$ is the mass flux of the $k^{\text {th }}$ species due to the electrical field only in a system without any chemical potential gradients. The quantity in the bracket in Eq. (9) is $t_{k}$, the transference number which can be measured by the Hitorff method in an independent experiment. The electrical transference numbers satisfy the following condition:

$$
\sum_{k=1}^{N} t_{k}=1 \quad \text { and } \quad t_{0}=0
$$

and are the measure of the easiness of migration of a particular ionic species in responding to the electrical field. Rewrite Eq. (9) in terms of $t_{k}$.

$$
\frac{\partial \psi}{\partial X}=-\sum_{j=1}^{N} \frac{t_{j}}{Z_{j} F} \frac{\partial \mu_{j}}{\partial X}
$$

Substituting Eq. (9a) for Eq. (6b)

$$
\begin{gathered}
J_{i}=-\sum_{k=1}^{N} L_{i k} \frac{\partial \mu_{k}}{\partial X}+\sum_{k=1}^{N} Z_{k} L_{i k} \sum_{j=1}^{N} \frac{t_{j}}{Z_{j}} \frac{\partial \mu_{j}}{\partial X} \\
i=1,2,3, \ldots \mathcal{N} .
\end{gathered}
$$

Exchange the indices $k$ and $j$ in the second term in the above equation.

$$
J_{i}=-\sum_{k=1}^{N} L_{i k} \frac{\partial \mu_{k}}{\partial X}+\sum_{j=1}^{N} Z_{j} L_{i j} \sum_{k=1}^{N} \frac{t_{k}}{Z_{k}} \frac{\partial \mu_{k}}{\partial X}
$$

or

$$
J_{i}=-\sum_{k=1}^{N}\left[L_{i k}-\frac{t_{k}}{Z_{k}}\left(\sum_{j=1}^{N} Z_{j} L_{i j}\right)\right] \frac{\partial \mu_{k}}{\partial X}
$$

or

$$
\begin{array}{r}
J_{i}=-\sum_{k=1}^{N}\left[L_{i k}\left(1-t_{k}\right)-\sum_{j \neq k}^{N-1} \frac{Z_{j}}{Z_{k}} t_{k} L_{i j}\right] \frac{\partial \mu_{k}}{\partial X} \\
i=1,2,3, \ldots \mathcal{N} . \ldots \ldots \ldots \ldots
\end{array}
$$

Equation (10) says that the contribution of the chemical potential gradient of the $k^{t h}$ species on the flux of the $i^{\text {th }}$ component consists of three parts. The direct interactions, chemically and electrically are represented by the terms of

$$
L_{i k} \frac{\partial \mu_{k}}{\partial X} \quad \text { and } \quad t_{k} L_{i k} \frac{\partial \mu_{k}}{\partial X}
$$

respectively. The indirect interactions, through the third body, are given as the remaining $\mathcal{N}-1$ terms in the equation. It is obvious that when the coupling is strong enough a flux can be in a direction against its own chemical potential, or concentration, gradient.

\section{Discussion}

The equilibrium state of the system may be obtained by letting all fluxes vanish. It is obvious from Eqs. (6a) and (6b), as

$$
J_{i}=0, \quad i=1,2,3, \ldots \mathcal{N}
$$

the following condition must be satisfied:

$$
X_{k}=-\frac{\partial \bar{\mu}_{k}}{\partial X}=0, \quad k=1,2,3, \ldots \mathcal{N}
$$

or

$$
\frac{\partial \mu_{k}}{\partial X}=-Z_{k} F \frac{\partial \psi}{\partial X}, \quad k=1,2,3, \ldots \mathcal{N}
$$

\footnotetext{
* Similar expression has been given by many authors for the derivation of liquid junction potential, for example, see REF. 5).
} 


$$
\begin{aligned}
-F \frac{\partial \psi}{\partial X} & =\frac{1}{Z_{k}} \frac{\partial \mu_{k}}{\partial X}=\frac{1}{Z_{i}} \frac{\partial \mu_{i}}{\partial X}, \\
i, k & =1,2,3, \ldots \mathcal{N} \ldots \ldots \ldots
\end{aligned}
$$

Equation (11) demands that, under equilibrium conditions, the gradients of the chemical potentials (or concentrations) are uniquely related. The partial equilibrium state, chemical potential (concentration) gradients for all species except a few under consideration, cannot be reached. To have a system which is uniform in composition, as the final state of a diffusing ionic system is a special case $(\partial \psi / \partial X=0)$ rather than the rule. Of course, a given gradient of the chemical potential of any particular species can be sustained under a no flux condition by an externally imposed field, however, the value for all the other species is also uniquely fixed according to Eq. (11).

In order to visualize its generality, Eq. (10) can be easily reduced to a simpler form which has been reported in the literature. The coupled diffusion of oxygen and sulfur ions through sodium glass diaphragm under well controlled boundary conditions was investigated by Turkdogan and Grieveson. ${ }^{6)}$ The proper interpretation of their results was given by Lu and $\mathrm{Jin}^{4)}$ in terms of the linear phenomenological equations. This system may be considered as a pseudo-ternary system with

$$
Z_{1}=Z_{2}=-2, \quad t_{3}=J_{3}=0 .
$$

Substituting these data into Eq. (10), then we have

$$
J_{1}=-J_{2}=-\left(L_{11} t_{2}-L_{12} t_{1}\right) \frac{\partial}{\partial X}\left(\mu_{1}-\mu_{2}\right) \ldots \ldots
$$

And from Eqs. (8) and (9a)

$$
J_{1}=-J_{2}=-\frac{L_{11} L_{22}-L_{12} L_{21}}{L_{11}+L_{21}+L_{22} L_{12}} \frac{\partial}{\partial X}\left(\mu_{1}-\mu_{2}\right) .
$$

For the case,

$$
L_{12} L_{21} \ll L_{11} L_{22}
$$

and

$$
L_{12}+L_{21} \ll L_{11}+L_{22} .
$$

Equation (13) becomes

$$
J_{1}=-J_{2}=-\frac{L_{11} L_{22}}{L_{11}+L_{22}} \frac{\partial}{\partial X}\left(\mu_{1}-\mu_{2}\right) .
$$

This is the equation derived ${ }^{4}$ by assuming $L_{i j}=0$ where $i \neq j$, in Eq. (6). The application of Eq. (10) in interpretating the data on sulfur transfer obtained by King et al. ${ }^{7)}$ with a new formalism of phase boundary slag/metal reactions will be given elsewhere. ${ }^{8)}$

It is worth nothing that Eq. (9), which expresses the electrical field in terms of $t$ 's rather than $L$ 's, helps in revealing the underlying physical meaning of the various terms in Eq. (10). Because the $t$ 's can be measured in an independent experiment, a complicated system such as the industrial slags may possibly be analyzed in terms of Eq. (10). Lu and Jin ${ }^{4)}$ carried out, in detail, the calculations in order to compare Eq. (14) and the experimental data of Turkdogan and Grieveson. The description of these steps will not be repeated here. Furthermore, Kirkaldy ${ }^{3)}$ recently reviewed the progress in the study of diffusion in multiple-component systems including the transformation of phenomenological equations like Eq. (10) into diffusion equations. It is considered that the aim of the present communication in presenting a new formalism is accomplished after obtaining Eq. (10).

\section{Acknowledgement}

The author wishes to thank the Research Board of the Division of Science and Engineering, McMaster University for financial support of this project and Mr. D. E. Coates for his advice.

\section{REFERENGES}

1) E. A. Guggenheim: J. Phys. Chem., 33 (1929), 842. E. A. Guggenheim: Thermodynamics, (1967), 298, North Holland.

2) S. R. de Groot: Thermodynamics of Irreversible Processes, (1952), 182, North-Holland.

3) J.S. Kirkaldy: Advances in Materials Research, 1969, (In Press).

4) W-K. Lu and I. Jin: Met. Trans., 1 (1970), 267

5) J. O'M. Bockris and W. K. N. Reddy: Modern Electrochemistry, (1970), 399, Plenum Press.

F. O. Koenig: J. Phys. Chem., 44 (1940), 101.

D. A. MacInnes: The Principles of Electrochemistry, (1939), 220 ff, Reinhold.

6) E. T. Turkdogan and P. Grieveson: Trans. Met. Soc. AIME, 224 (1962), 316.

7) S. Ramachandran, T. B. King, and N.J. Grant: Trans. Met. Soc. AIME, 206 (1956), 1546.

8) International Conference on the Science and Technology of Iron and Steel, held in Tokyo, Japan, September, 1970. 